illness due to diphtheria and 401 deaths from that disease if the 1890 ratio of prevalence and death had continued to prevail. Had the 1890 ratio of prevalence and death continued throughout the whole ten years, there would have occurred in the actual population of Denver during those years a total of 8993 cases of diphtheria, of whom 3450 would have died.

By the gradual operation of the preventive measures that have been perfected during these ten years, the actual number of cases of diphtheria was reduced to a total of 344.9 , or a saving of 5444 cases of illness from this one disease. The actual number of deaths from diphtheria has been reduced from the probable total of 3450 to an actual death-loss of 963 , or an actual saving of 2487 lives. Had we been able to apply the modern preventive methods of the last four years with an average per annum of but 294 cases and 34 deaths throughout the whole decade, the actual saving of human life would have been still greater, approximating 3200 .

This saving of human life and of actual human suffering has been the gift of regular scientific medicine to the communitty in which I live during the past decade in regard to the one disease, diphtheria, alone. It is a gift that has been duplicated in so far as actual lifesaving has been concerned in regard to typhoid fever. This public benefaction has been made possible only through the discoveries of modern medicine and their application by the regular medical profession in the interest of the public welfare. It has been forced by us on an unwilling and ignorant public whose individual members and official representatives thought that we were struggling for our own welfare instead of the public good. A lawyer whom the fortunes of politics landed in the position of Governor of Colorado has even had the assurance to place on the public records, in a message wherein he vetoed a medical practice act, the silly assertion that no advance in science for the public welfare had ever been made by the regular medical profession.

The great object-lesson of the diminution of diphtheria and typhoid fever in the community where he himself has been privileged by a merciful Providence to live for thirty long years has been altogether lost on him. We are sometimes so irritated by the existing order of things that we protest, like good old David, against the folly of those who are high in power. We ask ourselves, "Is it worth while to labor for the welfare of a blind and thankless people?"

The answer is older than holy writ. It lies within our own hearts and it must still be the inspiration for future work. Duty done is its own best reward. The plaudits of an ignorant world might make work easier, but can not make it more honorable; the crackling of thorns under the pot adds no heat to the fire.

\section{THE ASSOCIATE INFECTIONS OF SCARLET FEVERS.*}

BY W. J. JAQUES, M.D. CHICAGO.

The object of this paper is to call attention to the fact that scarlet fever is seldom a single infection, and its successful treatment necessitates a knowledge of all the factors contributing to the abnormal condition.

It has not yot been positively demoustrated, at least it is not generally accopted, that the causative agent of scarlet fever is a germ, although there is much evidence

*Presented in a Symposium on Diphtheria. berore the Section on State Medicine, at the Fiftieth Annual Meeting of the American Mecical Association, held at Columbus, Ohio, June 6-9, 1899. to indicate it. Each case seems to come from some other case, and under certain conditions it is known to be highly contagious. It also resembles other diseases which we know are caused by a germ. The difficulty of staining the tubercle bacillus delayed its identification. Some similar characteristic may be preventing the identification of the scarlet fever germ.

During the past winter, Dr. J. W. Class ${ }^{1}$, Chicago, called the attention of the medical world to a coccus that he found constantly present in the scales and mucus taken from scarlet fever patients. Its size is exceedingly variable. From a small dot hardly to be seen with a one-twelfth objective, it becomes three or four times as large as the gonococcus, which it resembles in shape. The size depends on the age of the culture, and the growth in the mouth is less than that on culturemedia. In growing, the cultures produce a gelatinous substance which is drawn out in strings with a platinum needle. It does not seem improbable that these cocci may enter the blood in their minute form, clogging and congesting the capillaries.

Since the description of this germ and the method of staining were given, I have taken cultures from all cases of scarlet fever coming under my care and found it uniformly present. I have also found the germ present in a small proportion of the cultures which $I$ examined for suspected diphtheria. In three cases of scarlet fever I was enabled to make the diagnosis of scarlet fever before any appearance of rash. I did this from the malignancy of the germ as indicated by the numbers and the rapidity of their growth. I have also found the germ present in the throats of adults and nurses who were taking care of patients with this disease. However, it takes the combined evidence of many observers to arrive at definite conclusions, and until this, or some other, germ is identified and enough of its characteristics are known to show us a better way to protect ourselves, we must rely on the knowledge and experience of the past.

Every advance made in scientific medicine must be based on an accurate knowledge of physiology. When the functions and conditions necessary for normal metabolism are understood, it will not be difficult to comprehend the causes of abnormal metabolism, or disease.

We should keep before us the importance of the individuality of the cell; that it is the basis of all forms of life from the minutest bacteria to the giant of the forest. All the higher forms of life are built up out of structural units similar to the simple bacterial cell.

The human body, for our present purpose, may be considered as made up of groups of individual cells, performing distinct as well as general functions. The pathogenic organism that attacks it is also a cell having like functions of absorption, excretion and reproduction. It becomes pathogenic when it finds in the human body suitable conditions where it can complete its cycle of activity.

The study of a germ disease, then, resolves itself into a study of the patient and those conditions which produce susceptibility; also a study of the germ and the environment which causes it to become pathogenic. A contagious discase may be likened to a union of two pieces of chain, the links of one piece, produced by a cycle of events in the germ, and the other by a cycle of events in the patient. The chain may be broken by breaking one of the links.

A germ is pathogenic only in the presence of a susceptible individual. The environment which causes susceptibility in a patient may also increase the virulence 
of the germ. During the winter months, when confinement and lack of sunshine causes a decrease in vital resistance, the virulence of the germs of diphtheria, pneumonia and influenza is greatly increased.

The whole phenomena of life is a battle of cells. Outside of age and hereditary influences, the survival of the fittest depends on environment. By changing the conditions of environment in the patient, we may prevent that part of the chain from forming. By changing the environment of the germ, the chain is broken there. No one contracts tuberculosis until a suitable soil is first produced on which the bacilli will grow. The cholera bacillus is pathogenic only in the presence of decomposing filth.

Susceptibility to many germ diseases may be caused by exposure, lack of nutrition, loss of rest, or any other factor which causes a lowering of physiologic resistance; but susceptibility to malaria and diphtheria may exist in health. In many fermentative processes the germs succeed each other, one preparing the way for a second. A person may be susceptible to but one disease and that may produce susceptibility to another. Phthisis often follows pneumonia. Sanarelli points out that the causative germ of yellow fever is the bacillus icteroides, which multiplies in the human body to a limited extent; that the toxin produced by it facilitates secondary infections of all kinds which cause death.

It is my belief that a similar condition exists in scarlet fever. A distinct contagious element enters the body and multiplies. To a greater or less degree it causes constitutional disturbance and characteristic erythema. The physiologic resistance of the patient is lowered in direct ratio to the virulence of the contagion. The Klebs-Loffer bacilli and pus germs infest the mouth and may remain there for long periods without causing disease. When from any cause the physiologic resistance becomes lowered, they invade the tissues. Thus, if a child contracts scarlet fever and other germs are present in the mouth, it is highly probable that secondary infection will follow.

Since our use of the microscope in the study of these infections, we are enabled to distinguish the clinical symptoms which accompany the invasion of many of these germs. In pure Klebs-Loeffler diphtheria there may be little pain and no temperature. . The presence of diphtheria may be completely covered up by the more pronounced symptoms of scarlet fever. Clinically, it is impossible to diagnose the presence of diphtheria with scarlet fever during the first thirty-six hours. If the rash appears at the end of this time, the doctor usually makes the diagnosis of scarlet fever, and is satisfied. In such a case, if death should occur, the most important factor in causing the death, namely the diphtheria toxin, may not have been recognized.

The most dangerous infection associated with scarlet fever is diphtheria. Unfortunately many of the textbooks speak of this as being rare. Rapid transit and commercial exchange have greatly aided the distribution of germs. A few years ago an epidemic would not spread beyond a single neighborhood during the period of susceptibility. Now the spread of the influenza bacilli from one and of the continent to the other is only a matter of hours. Every factor which affects the condition of mankind must change his relation to germ disease; and every new method of hygiene and sanitation must affect the environment of the germ; hence the constant change in germ diseases. The use of diphtheria antitoxin has had the effect of lessening the fear of the pub- lic, and less care is taken to isolate diphtheria patients; hence a wider dissemination of these bacilli.

"F. Ranke ${ }^{2}$ found diphtheria bacilli present in the throats of 53.7 per cent. of scarlet fever patients in Munich. Goodall ${ }^{3}$ made a bacteriological examination of 87 cases of scarlet fever admitted into the Eastern fever hospital. The examination was made immediately after admission. The diphtheria bacilli were found in 19 cases. They were' mild and would not have been recognized clinically as diphtheria. One of the cases subsequently developed post-scarlatinal diphtheria and so did four other cases in whom no bacilli were found on admission. Sverensen ${ }^{4}$ examined 154.7 scarlet fever patients on admission to the fever hospital at Copenhagen and found diphtheria bacilli in 2.5 per cent. Beggs ${ }^{5}$ found diphtheria bacilli in the throats of 51 out of 140 cases of scarlet fever examined." The spread of germs being such an uncertain factor would explain partly the difference in results of these observations.

While the Klebs-Loffler bacilli are comparatively rare, the pus germs are almost always present. The streptococcus pyogenes causes the most painful and lasting of the anginas. Most cases of scarlet fever, after the symptoms of the specific contagion abates, resolve themselves into streptococcus infections. The first infection leaves the patient susceptible to invasion and there is often a general infection of the tissues and glands of the buccal region. The inflammation also extends into the middle ear and injures the delicate bones of the auditory apparatus. The judicious use of antistreptococcic serum is of valuet in preventing such sequelx. Not every case of scarlet fever requires the serum. From the clinical symptoms of the patient, and the malignancy of the pus germs as indicated by the culture, the physician must decide as to treatment. In most cases death is preferable to a deaf and dumb child, and it is as important to prevent scarlet fever from reaching the suppurative process in the ear as it is to cure the disease.

During the season of its prevalence influenza may often be found associated with scarlet fever, but its toxin has not such fatal effects on children as on adults. Measles, mumps and other diseases of childhood are apt to follow or be found with scarlet fever, appearing either as primary or secondary infections.

It should not be inferred that in these associate infections each germ presents itself in its most malignant form, thereby increasing the severity of the cases many fold. Ustally the conditions for malignancy favor but one germ at a time, the rest appearing in a minor rôle until a change of conditions permits their more rapid development.

Proceeding on the belief that scarlet fever is nearly always accompanied or followed by other infections, with a similarity of clinical manifestations during the early stages, it must be apparent how important a part the microscope has come to play in a correct diagnosis. If it can be known just what germs are present and the degree of malignancy, as indicated by their growth, treatment may assume the initiative and defensive, or preventive form, with a more favorable prognosis than is possible with a delayed diagnosis.

The first step to be taken in the treatment of every angina, however mild it may appear, should be the taking of a culture. 'This should be follorved at intervals during the progress of the disease by other cultures, to show by the morphologic ehanges whether the infection is subsiding or increasing; also whether or not the conditions have been favorable to the growth of other germs. 
With the bacterial evidence well in hand, the clinical symptoms become clearer and more definite, consequently easier to control by treatment. There is no one line of treatment which will fit every case of scarlet fever for the very rason assumed in the beginning, that it is so often modified and complicated with other infections. The kidneys, heart and mervous structure must be closely watched. The larynx may become involved. Nutrition must be strictly maintained and the destruction of renal tissues prevented. 'The selective action of scarlet fever poison and the toxins of associate infections must be kept constantly in mind. The stupor resulting from scarlet fever poison is not so serious nor important as if it is caused by the Klebs-Lœfller bacilli. In the latter case a process of degeneration of the nerve center is instituted which is likely to continue; it may be slowly or rapidly, resulting fatally; if not at once, it may be days or weeks after apparent recovery. This is not so with stupor caused by simple scarlet fever infection.

The prognosis is more favorable when this mental condition is caused by the scarlet fever toxin, and this knowledge should inspire hope while life is present in the patient. As long as the heart beats, every means should be exhausted to sustain the strength. In one of my cases oxygen gas and artificial respiration for thirty minutes tided the child over the crisis of the disease.

In malignant and prolonged cases of scarlet fever, the assistance of one or more trained nurses is absolutely necessary. This training must include infectious diseases, which are not usually part of the hospital course.

Not only should the nurse be familiar with the different manifestations of scarlet fever and the best methods of meeting them, as well as the most effectual means of disinfection, but she should be reasonably satisfied of her own immunity. Neither nurse nor physician should be handicapped by a fear of the disease, as the successful issue of a case may require most strenuous efforts and even the constant presence of both for a time.

With all these conditions accepted and carried out, deaths from scarlet fever complications will probably not be eliminated, but they will be much reduced.

4310 Greenwood Avenue.

BIBLIOGRAPHY

1. Jourat April 8, 1899. p. 765

2. Munich Med. Woch., Bd. 42, 1896 .

3. Epidem. Soc. Trans. 189

1898

5. Met. Asylums Board's Report, 1895.

\section{WOULD SURGERY SUFFER IF IODOFORM WAS ABOLISHED ?*}

BY EDMUND C. BRUSH, A.M., M.D.

Member Ohio State Medical Society ; Member American Medical Asso. ciation; Consulting Surgeon to Zanesville City Hospital, etc. ZANESVILIE, OHIO.

You may break, you may shatter the box, if you will, But the scent of iodoform will linger there still.

If something else just as efficacious can be found, or is at hand, then iodoform should be forever banished, on account of its odor if for no other reason. This odor is unmistakable, and to-day the laity know it quite as well as the physician. "Once smelled, always remembered," is surely true of this promiscuously used drug. You meet a man on the street and, as he passes, you get a whiff of iodoform; you at once wonder whether he is a physician or a patient. You enter a sleeping-car and the well-known odor comes to your attention; you at once look over your fellow passengers for the sus-

*Presented to the Section on Surgery and Anatomy, at the Fiftieth Annual Meeting of the American Medical Association, held at Columbus, Ohio, June 6-9, 1899. pected one. You get the same odor in the ballroom or in the church, and you at once wonder who has it concealed about the person and why.

I am fully aware that the reign of iodoform is on the wane, and, hoping to add to its non-use I present the subject here. To get substantial data from which to draw conclusions and to give influence, I sent out a circular letter to representative surgeons in Great Britain, Canada and the United States. All of these letters went to gentlemen who hold surgical positions, a large majority being professors of surgery in medical colleges. Medical schools in London, Edinburgh, Dublin, Quebec, Toronto, Montreal and twenty-two of the United States are represented in the replies; so that the information is general and not in the least localized. The circular letter read as follows:

Doctor:-I am preparing for the coming meeting of the American Meidcal Association a paper entitled "Would Surgery Suffer if Iodoform Was Abolished?" In order to give weight, either for or against the question, I am writing to one hundred representative surgeons and asking them to answer the accompanying questions. Will you favor me by answering the questions and returning this letter in the inclosed envelope? sincerely yours, E. C. Brush.

The first question asked was: "What germicidal powder do you prefer? Of the 84 replying, 27 used iodoform, 24 used no powder of any kind, 12 boric acid, and 8 used aristol. The remaining 13 used acetanilid, nosophen, iodol, etc.

The second question was for second choice of powders, and the answers have no special significance.

The third question was: "Have you seen any bad effects from using iodoform? If so, what?" Seventythree report that they have seen bad effects, 11 have not, but 4 of the 11 have seen no bad effects because they did not use the drug.

The fourth question was: "Would, in your opinion, surgery suffer if iodoform was abolished?" Thirtyseven think that the drug could be abolished without any detriment to surgery, $4: 7$ that surgery would suffer. of these $4 \%, 2 \%$ think the detriment would be in a general way; 12 limit the loss to the treatment of surgical tuberculosis, and 8 consider the loss to be only in connection with its use in the manufacture of gauze, and in specific cases.

The third question: "Have you seen any bad effects from using iodoform? If so, what?" is the most important and the one bearing directly on the question at issue. Please remember that 73 out of 84 report bad effects, and these range from a slight local dermatitis to death; 28 report local dermatitis, 3 general dermatitis, and 5 report eruptions, and 20 iodoform poisoning without any qualification; 2 report delirium and collapse, 5 intoxication, 14 general systemic effects, 1 congestion and inflammation of the kidneys, 6 delirium, and 1 reports paralysis. Five deaths are reported.

The difference between the number of ailments reported and the number of gentlemen reporting them is due to the fact that several different surgeons report several different toxic effects. Then, too, it is evident that many of them, although using different words, probably mean the same thing. For instance, 20 mention iodoform poisoning and 14 general systemic effects. The two expressions are, no doubt, in a measure used synonymously. It will be noticed that about all of the known poisonous effects of iodoform are mentioned by the 75 surgeons who report them. It might be well to remember that $\% 3$ form a very small proportion of 